UCD-03-11

hep-th/0311090

\title{
Non-Quasinormal Modes and Black Hole Physics
}

\author{
Danny Birmingham ${ }^{*}$ and S. Carlin \\ Department of Physics, University of California, Davis, CA 95616
}

(Dated: October 31, 2018)

\begin{abstract}
The near-horizon geometry of a large class of extremal and near-extremal black holes in string and $\mathrm{M}$ theory contains three-dimensional asymptotically anti-de Sitter space. Motivated by this structure, we are led naturally to a discrete set of complex frequencies defined in terms of the monodromy at the inner and outer horizons of the black hole. We show that the correspondence principle, whereby the real part of these "non-quasinormal frequencies" is identified with certain fundamental quanta, leads directly to the correct quantum behavior of the near-horizon Virasoro algebra, and thus the black hole entropy. Remarkably, for the rotating black hole in five dimensions we also reproduce the fractionization of conformal weights predicted in string theory.
\end{abstract}

PACS numbers: 04.70.Dy,11.25.Hf,11.25.Uv

\section{INTRODUCTION}

It is generally expected that a thorough understanding of the quantum mechanical nature of black holes will help pave the way towards a fully consistent theory of quantum gravity. In this context, one recent effort has focused on applying the correspondence principle to black hole physics. The central idea [1, 2] is to identify the real part of the classical quasinormal frequencies of gravitational perturbations with the elementary quanta of black hole mass and angular momentum. Interest in such a proposal increased with the observation [3] that such a correspondence can correctly fix the Immirzi parameter [4], an undetermined prefactor in the area operator of loop quantum gravity. However, a general understanding of the universality of such an approach is still lacking. In particular, the applicability to charged and rotating black holes remains unclear; see for example [5, 6, 7, 8, 9, 10, 11].

In 12], we studied the correspondence principle as it applies to the $(2+1)$-dimensional black hole of Bañados, Teitelboim, and Zanelli (BTZ) [13]. The quantum mechanics of the BTZ black hole is characterized by a Virasoro algebra. By identifying the real part of the quasinormal modes with the fundamental quanta of black hole mass and angular momentum, we found that an elementary excitation corresponds exactly to a correctly quantized shift of the Virasoro generators $L_{0}$ and $\bar{L}_{0}$ of this conformal algebra. Furthermore, we showed that by applying the correspondence principle to the mass and angular momentum parameters of point particle spacetimes and their analogs in Liouville theory [14], one can also account for transitions among ground states whose values of $L_{0}$ and $\bar{L}_{0}$ do not differ by integers.

In this paper, we consider general classes of four- and five-dimensional near-extremal, charged, rotating black holes that arise in string and M theory [15, 16, 17]. The near-extremal Reissner-Nordström black hole occurs as a special case. Although these black holes are asymptotically flat, they have the special feature that the geometry in the near-horizon region contains that of the BTZ black hole [18, 19]. In particular, it has been shown that the associated near-horizon conformal symmetry leads directly to a microscopic understanding of the BekensteinHawking entropy 19, 20, 21, 22]. Our aim is to understand how the correspondence principle might be applied to these higher-dimensional black holes to capture the correct quantization of this symmetry.

Conventionally, quasinormal mode boundary conditions are stated in terms of behavior of perturbations at the horizon and infinity. This has always been somewhat mysterious, since it is not clear why black hole quantization should care about infinity. Here, we first show that for the BTZ black hole, these boundary conditions can be recast in terms of monodromy conditions at the inner and outer horizons 23. Armed with this knowledge, we are led naturally to define a set of "non-quasinormal modes" for the higher-dimensional black holes involving only these monodromies. The correspondence principle leads directly to the correct quantization of the nearhorizon Virasoro generators. Remarkably, for the rotating black hole in five dimensions, the correspondence principle also leads to the correct fractionization of conformal weights, in exact agreement with the predictions of a microscopic D-brane analysis [24, 25, 26].

\section{HORIZON MONODROMIES}

For black holes in asymptotically anti-de Sitter spacetimes, the quasinormal modes are defined as solutions that are ingoing at the horizon and either vanish [27] or have vanishing flux [28] at infinity. Let us first consider the quasinormal modes of the BTZ black hole, which is a solution of the vacuum Einstein equations in three spacetime dimensions with negative cosmological constant $\Lambda=-1 / l^{2}$. The black hole is parametrized by its ADM mass $M_{B T Z}$ and angular momentum $J_{B T Z}$, and has inner and outer horizons at $\rho_{ \pm}$, with

$$
8 G_{3} M_{B T Z}=\frac{\rho_{+}^{2}+\rho_{-}^{2}}{l^{2}}, \quad 8 G_{3} J_{B T Z}=\frac{2 \rho_{+} \rho_{-}}{l} .
$$


For simplicity, we consider the quasinormal modes of an infinitesimal 36] massless scalar perturbation $\Phi$ satisfying $\nabla^{2} \Phi=0$. The solution can be written as

$$
\Phi=R(\rho) \exp \left\{-i \omega_{B T Z} t_{B T Z}+i k_{B T Z} \phi_{B T Z}\right\},
$$

where the radial function satisfies the hypergeometric equation with regular singular points at the inner and outer horizons and infinity [30, 31]. Near the outer horizon, the radial part of the ingoing solution takes the form

$$
R\left(\rho_{+}\right) \sim\left(\rho^{2}-\rho_{+}^{2}\right)^{-\frac{i}{8 \pi}\left[\beta_{R}\left(\omega^{B T Z}+\frac{k^{B T Z}}{l}\right)+\beta_{L}\left(\omega^{B T Z}-\frac{k^{B T Z}}{l}\right)\right]},
$$

with $\beta_{R, L}=2 \pi l^{2} /\left(\rho_{+} \pm \rho_{-}\right)$; the inverse temperatures at the inner and outer horizons are $\beta_{ \pm}=\left(\beta_{R} \pm \beta_{L}\right) / 2=$ $-\Omega_{\mp} \beta_{\mp} l$, where $\Omega_{ \pm}$are the angular velocities of the inner and outer horizons. Inspired by [5, 23], we consider the monodromy of this solution - the change under a $2 \pi$ rotation in the complex $\rho$ plane around the zero at $\rho=\rho_{+}-$ which is given by

$$
\begin{aligned}
& \mathcal{M}\left(\rho_{+}\right)= \\
& \quad \exp \left\{\frac{1}{4}\left[\beta_{R}\left(\omega^{B T Z}+\frac{k^{B T Z}}{l}\right)+\beta_{L}\left(\omega^{B T Z}-\frac{k^{B T Z}}{l}\right)\right]\right\} .
\end{aligned}
$$

The conventional way to determine the quasinormal modes is to continue this solution to infinity and demand that it satisfy Dirichlet boundary conditions. We employ an alternative procedure. We first continue the ingoing solution to the inner horizon, where it becomes a particular linear combination of the two independent solutions at $\rho_{-}$, with behaviors that follow from standard properties of hypergeometric functions:

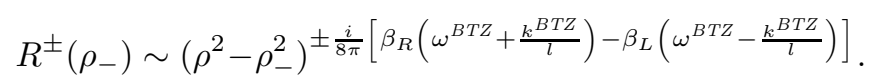

The monodromies of these solutions around $\rho=\rho_{-}$are

$$
\begin{aligned}
& \mathcal{M}^{ \pm}\left(\rho_{-}\right)= \\
& \exp \left\{ \pm \frac{1}{4}\left[\beta_{R}\left(\omega^{B T Z}+\frac{k^{B T Z}}{l}\right)-\beta_{L}\left(\omega^{B T Z}-\frac{k^{B T Z}}{l}\right)\right]\right\} .
\end{aligned}
$$

We now demand that the product of monodromies at the inner and outer horizons be trivial, $\mathcal{M}\left(\rho_{+}\right) \mathcal{M}\left(\rho_{-}\right)=1$. It may be checked that this condition is satisfied if either $\mathcal{M}\left(\rho_{+}\right) \mathcal{M}^{+}\left(\rho_{-}\right)=1$ or $\mathcal{M}\left(\rho_{+}\right) \mathcal{M}^{-}\left(\rho_{-}\right)=1$, leading to frequencies (for $\operatorname{Im} \omega^{B T Z}<0$ )

$\omega_{L}^{B T Z}=\frac{k_{L}^{B T Z}}{l}-\frac{4 \pi i}{\beta_{L}}(n+1), \quad \omega_{R}^{B T Z}=\frac{k_{R}^{B T Z}}{l}-\frac{4 \pi i}{\beta_{R}}(n+1)$,

where the mode number $n \in \mathbf{N}$, and $k_{L}, k_{R} \in \mathbf{Z}$. These frequencies are immediately recognized as the quasinormal modes of the BTZ black hole [30, 31]. While the monodromy condition involves the behavior of the perturbation only in the region between the inner and outer horizon, it is nevertheless equivalent to the conventional quasinormal mode boundary conditions involving behavior outside the outer horizon. This reinterpretation has been discussed in the static case in [23].

\section{CHARGED 4-DIMENSIONAL BLACK HOLES}

We now consider a class of four-dimensional charged black holes arising in M theory 15, 16]. For our purposes, it is convenient to reinterpret these as five-dimensional black string solutions by implementing a boost along a fifth direction 20]. The line element then takes the form

$$
\begin{aligned}
d s^{2}= & \left(H_{1} H_{2} H_{3}\right)^{-\frac{1}{3}}\left[-f d \tilde{t}^{2}+d \tilde{x}^{2}\right] \\
& +\left(H_{1} H_{2} H_{3}\right)^{\frac{2}{3}}\left[f^{-1} d r^{2}+r^{2} d \Omega_{2}^{2}\right],
\end{aligned}
$$

where $f=1-r_{0} / r, \tilde{t}=t \cosh \delta_{0}-x \sinh \delta_{0}$, and $\tilde{x}=-t \sinh \delta_{0}+x \cosh \delta_{0}$. The harmonic functions $H_{i}$ are $H_{i}=1+\frac{r_{0} \sinh ^{2} \delta_{i}}{r}$, and the physical charges are $Q_{i}=\frac{1}{2} r_{0} \sinh 2 \delta_{i}$. The general solution depends on five parameters $r_{0}, \delta_{0}, \delta_{1}, \delta_{2}, \delta_{3}$; the case $\delta_{0}=\delta_{1}=\delta_{2}=\delta_{3}$ is the standard Reissner-Nordström black hole.

We wish to study a perturbation of the black string by a massless scalar field $\Phi$ satisfying $\nabla^{2} \Phi=0$. Using the ansatz $\Phi=e^{-i \tilde{\omega} \tilde{t}+i \tilde{k} \tilde{x}} R(r) Y(\theta, \phi)$, the full fivedimensional wave equation can be written down explicitly. For our discussion, we need only consider the leading terms close to the horizons, namely [32]

$$
\begin{aligned}
\frac{d}{d x}\left[\left(x^{2}-\frac{1}{4}\right) \frac{d R}{d x}\right] \\
+\frac{1}{x-\frac{1}{2}}\left(\tilde{\omega} r_{0} \cosh \delta_{1} \cosh \delta_{2} \cosh \delta_{3}\right)^{2} R \\
\quad-\frac{1}{x+\frac{1}{2}}\left(\tilde{k} r_{0} \sinh \delta_{1} \sinh \delta_{2} \sinh \delta_{3}\right)^{2} R=0,
\end{aligned}
$$

where we have introduced the coordinate $x=\frac{r}{r_{0}}-\frac{1}{2}$.

As usual, we first select a solution that is ingoing near the outer horizon $x=\frac{1}{2}$. This has the form

$$
R \sim\left(x-\frac{1}{2}\right)^{-i \tilde{\omega} r_{0} \cosh \delta_{1} \cosh \delta_{2} \cosh \delta_{3}} .
$$

The general solution near the inner horizon $x=-\frac{1}{2}$ is

$$
\begin{aligned}
R \sim A_{+} & \left(x+\frac{1}{2}\right)^{i \tilde{k} r_{0} \sinh \delta_{1} \sinh \delta_{2} \sinh \delta_{3}} \\
& +A_{-}\left(x+\frac{1}{2}\right)^{-i \tilde{k} r_{0} \sinh \delta_{1} \sinh \delta_{2} \sinh \delta_{3}} .
\end{aligned}
$$

In the dilute gas regime $\delta_{1}, \delta_{2}, \delta_{3} \gg 1$, the demand that the product of the monodromies at the two horizons be trivial then determines the frequencies (with $\operatorname{Im} \omega<0$ )

$$
\omega \pm k=-\frac{8(n+1) i}{r_{0}} \exp \left\{-\left(\delta_{1}+\delta_{2}+\delta_{3}\right) \pm \delta_{0}\right\}
$$


where $\omega=\tilde{\omega} \cosh \delta_{0}+\tilde{k} \sinh \delta_{0}, k=\tilde{\omega} \sinh \delta_{0}+\tilde{k} \cosh \delta_{0}$, and $n \in \mathbf{N}$. Note that the dilute gas condition implies that the black hole is necessarily near-extremal.

To make contact with our previous discussion, recall that the geometry in the near-horizon limit takes the form BTZ $\times S^{2}$, with the mass and charge of the black string reinterpreted in terms of the mass and angular momentum of the BTZ black hole 18, 19, 20. Specifically, one finds the identification of coordinates [20]

$$
t_{\text {BTZ }}=\frac{t l}{R_{x}}, \quad \phi_{\text {BTZ }}=\frac{x}{R_{x}}, \quad \rho^{2}=\frac{2 R_{x}^{2}}{l}\left(r+r_{0} \sinh ^{2} \delta_{0}\right),
$$

with effective three-dimensional mass and angular momentum parameters

$8 G_{3} M_{B T Z}=\frac{2 r_{0} R_{x}^{2}}{l^{3}} \cosh 2 \delta_{0}, \quad 8 G_{3} J_{B T Z}=\frac{2 r_{0} R_{x}^{2}}{l^{2}} \sinh 2 \delta_{0}$,

where $R_{x}$ is the radius of the compact $x$-direction. The three-dimensional cosmological constant is $\Lambda=-1 / l^{2}$, with $l=2\left(Q_{1} Q_{2} Q_{3}\right)^{\frac{1}{3}}$, and the gravitational coupling is $G_{3}=2 R_{x} G_{4} / l^{2}$. Using the identifications (13) and (14) and the dilute gas condition $\delta_{1}, \delta_{2}, \delta_{3} \gg 1$, one can check that the frequencies (12) agree precisely with (7).

It is important to stress that the black string solution is asymptotically flat, and conventional quasinormal mode boundary conditions would demand an outgoing solution at infinity. Our monodromy condition, however, requires that either $A_{+}=0$ or $A_{-}=0$, and is incompatible with such boundary conditions. We shall call our novel set of frequencies "non-quasinormal modes."

(Further "stringy" information can be obtained from purely ingoing modes used to compute greybody factors 33], but these require different boundary conditions, and in particular lead to real, nonquantized frequencies.)

We can now apply the correspondence principle, identifying the real part of these frequencies with certain fundamental quanta. The key is the presence of a near-horizon conformal symmetry. The associated Virasoro generators can be expressed in terms of the effective threedimensional mass and angular momentum (14) as [20]

$$
\begin{aligned}
& L_{0}=\frac{1}{2}\left(M_{B T Z} l+J_{B T Z}\right)+\frac{l}{16 G_{3}}=\frac{R_{x}}{2}(E+P)+\frac{l}{16 G_{3}}, \\
& \bar{L}_{0}=\frac{1}{2}\left(M_{B T Z} l-J_{B T Z}\right)+\frac{l}{16 G_{3}}=\frac{R_{x}}{2}(E-P)+\frac{l}{16 G_{3}},
\end{aligned}
$$

where $E=\frac{r_{0}}{8 G_{4}} \cosh 2 \delta_{0}$ and $P=\frac{r_{0}}{8 G_{4}} \sinh 2 \delta_{0}$ are the energy and momentum of the black string flowing in the $x$-direction. Following [12], we identify the real part of the non-quasinormal modes with the fundamental quanta of $M_{B T Z}$ and $J_{B T Z}$, via

$$
\begin{aligned}
& \Delta M_{B T Z}=\omega_{L}^{B T Z}+\omega_{R}^{B T Z}=\frac{k_{L}^{B T Z}}{l}+\frac{k_{R}^{B T Z}}{l}, \\
& \Delta\left(\frac{J_{B T Z}}{l}\right)=\omega_{L}^{B T Z}-\omega_{R}^{B T Z}=\frac{k_{L}^{B T Z}}{l}-\frac{k_{R}^{B T Z}}{l} .
\end{aligned}
$$

This leads directly to quantization of the Virasoro operators, $\Delta L_{0}=k_{L}^{B T Z}$ and $\Delta \bar{L}_{0}=k_{R}^{B T Z}$. As a result, we obtain the correct quantization of the energy and momentum of the black string,

$$
\Delta E=\frac{1}{R_{x}}\left(k_{L}^{B T Z}+k_{R}^{B T Z}\right), \quad \Delta P=\frac{1}{R_{x}}\left(k_{L}^{B T Z}-k_{R}^{B T Z}\right) .
$$

\section{CHARGED ROTATING 5-DIMENSIONAL BLACK HOLES}

We next consider a class of five-dimensional charged rotating black holes that arise in M theory [17]. Our aim is to explore the new features coming from the rotating nature of the black hole. As above, these configurations can be reinterpreted as black strings, now in six dimensions. The general solution depends on six parameters [21] - a mass $m$, charges $Q_{1}=m \sinh 2 \delta_{1}, Q_{2}=m \sinh 2 \delta_{2}$, angular momentum parameters $l_{1}, l_{2}$, and a boost parameter $\delta_{0}$ along the compact string direction $y$. The system has a microscopic interpretation in terms of D-branes, charged with respect to $Q_{1}$ and $Q_{2}$ : it corresponds to a collection of $n_{1}$ D1-branes and $n_{2}$ D5-branes, with $n_{1}=\frac{\pi}{4} \frac{g \alpha^{\prime} Q_{1}}{G_{5} R_{y}}$ and $n_{2}=\frac{Q_{2}}{g \alpha^{\prime}}$, where $g$ and $\alpha^{\prime}$ are the string coupling and Regge slope and $R_{y}$ is the radius in the $y$ direction.

The near-horizon geometry is of the form $\mathrm{BTZ} \times S^{3}$, with effective three-dimensional mass, angular momentum, gravitational and cosmological constants 21]

$$
\begin{aligned}
8 G_{3} M_{B T Z} & =\frac{R_{y}^{2}}{l^{4}}\left[\left(2 m-l_{1}^{2}-l_{2}^{2}\right) \cosh 2 \delta_{0}+2 l_{1} l_{2} \sinh 2 \delta_{0}\right], \\
8 G_{3} J_{B T Z} & =\frac{R_{y}^{2}}{l^{3}}\left[\left(2 m-l_{1}^{2}-l_{2}^{2}\right) \sinh 2 \delta_{0}+2 l_{1} l_{2} \cosh 2 \delta_{0}\right] \\
l & =\left(Q_{1} Q_{2}\right)^{1 / 4}, \quad G_{3}=R_{y} G_{5} / \pi l^{3} .
\end{aligned}
$$

As before, we can study the full six-dimensional wave equation to look for the solutions near the inner and outer horizons. Using the identifications (18), we again find that the non-quasinormal frequencies are given by (7).

Using (18), the Virasoro generators take the form [21]

$$
\begin{aligned}
& L_{0}=\frac{R_{y}}{2}(E+P)-\frac{1}{n_{1} n_{2}} j_{L}^{2}+\frac{n_{1} n_{2}}{4}, \\
& \bar{L}_{0}=\frac{R_{y}}{2}(E-P)-\frac{1}{n_{1} n_{2}} j_{R}^{2}+\frac{n_{1} n_{2}}{4},
\end{aligned}
$$

where the energy $E$, momentum $P$, and angular momenta $j_{L, R}$ of the black string are

$$
\begin{aligned}
& E=\frac{\pi}{4 G_{5}} m \cosh 2 \delta_{0}, \quad P=\frac{\pi}{4 G_{5}} m \sinh 2 \delta_{0}, \\
& j_{L, R}=\frac{\pi}{16 G_{5}}\left(l_{1} \mp l_{2}\right) m e^{ \pm \delta_{0}+\delta_{1}+\delta_{2}},
\end{aligned}
$$

and the Virasoro algebra has central charge $c=6 n_{1} n_{2}$.

In the absence of angular momentum, the correspondence principle (16) again leads to the correct integer 
shifts in the energy and momentum of the string. To understand the angular momentum contribution in (19), we recall that the microscopic states of the BTZ black hole can be described by Liouville theory [34, 35], and that Liouville theory contains additional states whose values of $L_{0}$ and $\bar{L}_{0}$ agree precisely with those of point particle spacetimes in $2+1$ dimensions [14]. The Virasoro generators in this case are given by

$$
\begin{aligned}
& L_{0}=-\frac{l}{16 G_{3}}\left[1-4 G_{3}\left(m+\frac{j}{l}\right)\right]^{2}+\frac{l}{16 G_{3}}, \\
& \bar{L}_{0}=-\frac{l}{16 G_{3}}\left[1-4 G_{3}\left(m-\frac{j}{l}\right)\right]^{2}+\frac{l}{16 G_{3}} .
\end{aligned}
$$

By applying the correspondence principle to $m$ and $j$, via $\Delta\left(m+\frac{j}{l}\right)=2 k_{L}^{B T Z} / l$ and $\Delta\left(m-\frac{j}{l}\right)=2 k_{R}^{B T Z} / l$, one is led to quantization of the form 12 .

$\Delta L_{0}=k_{L}^{B T Z}-\frac{1}{n_{1} n_{2}}\left(k_{L}^{B T Z}\right)^{2}, \quad \Delta \bar{L}_{0}=k_{R}^{B T Z}-\frac{1}{n_{1} n_{2}}\left(k_{R}^{B T Z}\right)^{2}$.

Remarkably, this fractionization of conformal weights is in precise agreement with the results of the D-brane analysis [24, 25, 26]. A similar fractionization occurs in the four-dimensional rotating solution 22 .

\section{DISCUSSION}

In the absence of a complete quantum theory of gravity, it is natural to speculate that classical frequencies might be related to quantum energy levels. Our results have confirmed this intuition for a large class of "stringy" black holes, demonstrating that many features of quantization - even those as special as fractionization of conformal weights - fit such a correspondence principle. On the other hand, the modes we have considered are not the usual quasinormal modes, but rather a new set picked out by behavior near the inner and outer horizons. In one sense, this is physically sensible: black hole quantization ought to depend on properties at the horizon, not those at infinity, and many would find the symmetric appearance of the inner and outer horizons appealing. On the other hand, our results further deepen the mystery of why ordinary quasinormal modes, and not our "non-quasinormal modes," seem to be so closely related to the Immirzi parameter of loop quantum gravity. There is clearly much more to be understood.

\section{ACKNOWLEDGMENTS}

D.B. is grateful to the Departments of Physics at UC Davis and University of the Pacific for hospitality. S.C. was supported in part by U.S. Department of Energy grant DE-FG03-91ER40674.
* birm@itp.stanford.edu on leave from Dept. of Mathematical Physics, University College Dublin, Ireland $\dagger$ carlip@dirac.ucdavis.edu

[1] J. D. Bekenstein, in Proceedings of the Eight Marcel Grossmann Meeting on General Relativity, eds. T. Piran and R. Ruffini (World Scientific, Singapore 1999).

[2] S. Hod, Phys. Rev. Lett. 81 (1998) 4293.

[3] O. Dreyer, Phys. Rev. Lett. 90 (2003) 081301.

[4] G. Immirzi, Nucl. Phys. Proc. Suppl. 57 (1997) 65.

[5] L. Motl and A. Neitzke, Adv. Theor. Math. Phys. 7 (2003) 307.

[6] S. Hod, Phys. Rev. D67 (2003) 081501.

[7] E. Berti and K. D. Kokkotas, Phys. Rev. D68 (2003) 044027.

[8] E. Berti, V. Cardoso, K. D. Kokkotas, and H. Onozawa, hep-th/0307013

[9] S. Hod, gr-qc/0307060

[10] J. Oppenheim, gr-qc/0307089

[11] S. Musiri and G. Siopsis, hep-th/0309227

[12] D. Birmingham, S. Carlip, and Y. Chen, Class. Quant. Grav. 20 (2003) L239.

[13] M. Bañados, C. Teitelboim, and J. Zanelli, Phys. Rev. Lett. 69 (1992) 1849.

[14] Y. Chen, hep-th/0310234

[15] M. Cvetič and D. Youm, hep-th/9508058

[16] G. T. Horowitz, D. A. Lowe, and J. M. Maldacena, Phys. Rev. Lett. 77 (1996) 430.

[17] M. Cvetič and D. Youm, Nucl. Phys. B 476 (1996) 118.

[18] S. Hyun, J. Korean Phys. Soc. 33 (1998) S532.

[19] K. Sfetsos and K. Skenderis, Nucl. Phys. B517 (1998) 179 .

[20] V. Balasubramanian and F. Larsen, Nucl. Phys. B 528 (1998) 229.

[21] M. Cvetič and F. Larsen, Nucl. Phys. B 531 (1998) 239.

[22] M. Cvetič and F. Larsen, Phys. Rev. Lett. 82 (1999) 484.

[23] S. Musiri and G. Siopsis, hep-th/0308196

[24] J. C. Breckenridge, R. C. Myers, A. W. Peet, and C. Vafa, Phys. Lett. B 391 (1997) 93.

[25] J. C. Breckenridge, D. A. Lowe, R. C. Myers, A. W. Peet, A. Strominger, and C. Vafa, Phys. Lett. B 381 (1996) 423.

[26] J. Maldacena and L. Susskind, Nucl. Phys. B 475 (1996) 679 .

[27] G. T. Horowitz and V. E. Hubeny, Phys. Rev. D62 (2000) 024027.

[28] D. Birmingham, I. Sachs, and S. N. Solodukhin, Phys. Rev. Lett. 88 (2002) 151301.

[29] A. Ori, Phys. Rev. Lett. 68 (1992) 2117.

[30] D. Birmingham, Phys. Rev. D64 (2001) 064024.

[31] V. Cardoso and J. P. S. Lemos, Phys. Rev. D63 (2001) 124015.

[32] M. Cvetič and F. Larsen, Nucl. Phys. B506 (1997) 107.

[33] O. Aharony et al., Phys. Rep. 323 (2000) 183, sec. 5.6.

[34] O. Coussaert, M. Henneaux, and P. van Driel, Class. Quant. Grav. 12 (1995) 2961.

[35] K. Bautier, F. Englert, M. Rooman, and Ph. Spindel, Phys. Lett. B479 (2000) 291.

[36] Finite perturbations may destabilize the inner horizon 29]; for now, we evade this issue by considering only infinitesimal "test" fields to probe the black hole structure. 